Bull. Soc. Chim. Belg., 60, pp. 410-423, 4 fig., 1951

\title{
VI. La polydispersité de l'acide ribonucléique de la levure
}

\author{
par \\ V. DESREUX et J.M. GHUYSEN (Liège)
}

\begin{abstract}
SUmmary. - Yeast ribonucleic acid has been purified by fractional extraction at two $\mathrm{pH}$ and is undoubtedly heterogeneous. Fractions of much higher molecular weight than previously reported have been obtained and the higher molecular weight fraction $\gtrless 50.000)$ is stable at low temperature in the dry state. Many fractions obtained from crude yeast ribonucleic acid have different solubilities but more or less the same average molecular weight. This fact probably explains why the samples isolated by different workers using different precipitation methods, all fall in the same limits of molecular weights.

The fractions obtained by repeated precipitation are more heterogeneous than those obtained by systematic extraction.
\end{abstract}

\section{I. - InTroduction.}

Des recherches effectuées depuis plusieurs années au Laboratoire de Chimie-Physique de Liège $\left({ }^{1},{ }^{2},{ }^{3},{ }^{4},{ }^{5}\right)$ ont montré que l'acide pentose nucléique, isolé à partir de levure par la soude, était hétérogène $\left({ }^{3}\right)$ et que les courbes de solubilité qui avaient été obtenues il y a quelques années $\left({ }^{1},{ }^{2}\right)$ n'étaient qu'apparemment caractéristiques d'une substance homogène $\left({ }^{3}, 4\right)$. Dès que l'on augmentait la solubilité de cet acide en modifiant un des paramètres du système solvant-précipitant $(\mathrm{pH}$, force ionique, pourcentage d'alcool méthylique) on mettait en évidence l'hétérogénéité de la fraction soumise à l'analyse, toutes les courbes ainsi obtenues étant caractéristiques des solutions solides.

\footnotetext{
(1) H. Chantrenne : Bull. Soc. Chim. Belg., 55, 5 (1946),

(2) H. Chantrenne : Bull. Soc. Chim. Belg., 55, 118 (1946).

(3) J.M. GhuYsen : Bull. Soc. Chim. Belg., 59, 490 (1950).

(4) L. DeflCambe : Bull. Soc. Chim. Belg., 59, 508 (1950).

$\left({ }^{5}\right)$ L. Delcambe et V. Dessreux : Bull. Soc. Chim. Belg., 59, 521 (1950).
} 
D'autre part les valeurs des poids moléculaires des acides pentose-nucléiques préparés à partir de levure, de Bacillus Coli et de pancréas étaient très voisines (environ $15.000)\left({ }^{1}\right)$ mais notablement plus élevées que celles obtenues par d'autres auteurs qui le plus souvent n'avaient utilisés pour ce calcul que les données de diffusion assumant que les molécules étaient sphériques. Ce n'est que récemment que quelques résultats plus complets ont été publiés à ce sujet notamment par E. Volkin et C. E. Carter $\left({ }^{2}\right)$.

Les constantes de sédimentation trouvées par ces auteurs sont du même ordre de grandeur que celles trouvées par nous pour des fractions caractérisées par des courbes de solubilité typiques de solutions solides.

Il était donc nécessaire de rechercher si cette hétérogénéité était fonction d'une modification de masse, de charge ou de structure chimique. Afin de résoudre ce problème, nous devions disposer de quantités suffisantes de différentes fractions et la mise au point d'une technique de fractionnement plus rentable et plus efficace que celles utilisées jusque maintenant, a donc constitué la première partie de ce travail. Nous avons ensuite recherché les variations de la masse moléculaire en fonction de la solubilité pour les différentes fractions obtenues au cours de ce travail.

\section{II. - Fractionnement par extraction fractionnéE.}

De l'examen des courbes de solubilité publiées antérieurement, on pouvait prévoir que, par des fractionnements répétés par précipitation, on devait obtenir des échantillons d'acide pentose nucléique de solubilité de plus en plus faible et donc vraisemblablement de poids moléculaire de plus en plus élevé. Malbeureusement les rendements en produits très peu solubles furent toujours très faibles; en effet, si la séparation des fractions $A$ (peu soluble) et $B$ (très soluble) de Chantrenne est aisée, l'obtention en quantité suffisante d'échantillons d'acide nucléique de solubilité décroissante à partir de la fraction $\mathrm{A}$, est malaisée.

J. M. Ghuysen a mis en évidence l'action solubilisante

(1) L.Delcambe et V.Desseux, Bull. Soc. Chim. Belg. 59, 521, (1950).

(2) E. Volkin and C.E. CARTER : J. Am. Chem. Soc., 73, 1516 (1951). 
dans certaines conditions des produits de dégradation sur les grosses molécules d'acide nucléique. Lors de la précipitation fractionnée, la solution reste riche en ces molécules de faibles poids moléculaires jouant un rôle perturbateur, jusqu'à la fin de l'opération. C'est pour ces raisons que nous avons pensé appliquer à ce fractionnement, la technique de l'extraction fractionnée misè au point par l'un de nous ${ }^{1}$ ) dans le cas des polymères.

Rappelons brièvement la méthode; la substance à fractionner est dispersée par évaporation de sa solution sur un support inerte $(0,5$ grs de produits pour 100 grs de sable fin et lavé).

L'ensemble est introduit dans une colonne en verre maintenue à une température constante et inférieure à $10^{\circ} \mathrm{C}$; on lave ensuite la colonne à l'aide d'un système solvantprécipitant de composition variable. La variation continue de composition (c'est-à-dire du $\gamma$ ou $\frac{\text { volume précipitant }}{\text { volume total }}$ ) peut être réalisée á l'aide d'un des appareils décrits antérieurement. Enfin les différentes solutions obtenues peuvent être recueillies dans l'appareil collecteur automatique décrit également. Au cours de cette opération on élimine donc graduellement, dès le début de l'opération, les petites molécules très solubles qui, comme nous l'avons rappelé, perturbent le fractionnement des acides nucléiques peu solubles.

Remarquons d'autre part que lors de l'écoulement du liquide extracteur au travers de la colonne, on n'obtient vraisemblablement pas l'équilibre entre les deux phases en présence et que la vitesse de diffusion doit jouer un rôle dans ce fractionnement et le favoriser. La technique de l'extraction en système continu a été utilisée dans le but de fournir des résultats semi-quantitatifs permettant de prévoir les conditions optima d'une extraction discontinue effectuée sur une quantité plus importante de matière.

a) Extraction à $p H 3,5$.

Le solvant est constitué d'un tampon acétate de $\mathrm{pH} 3,5$ et de force ionique égale à 0,10 . Le précipitant contient de

(1) V. Desneux : Rec. Trav. Chim. Pays-Bas, 68, 789 (1949). 
sur les

itation faibles qu'à la avons de l'ex1) dans

à fracsur un able fin

verre $10^{\circ} \mathrm{C}$; olvantinue de ) peut rement. recueillement. lement, is solue frac-

lent du obtient ases en un rôle de l'exbut de prévoir ffectuée

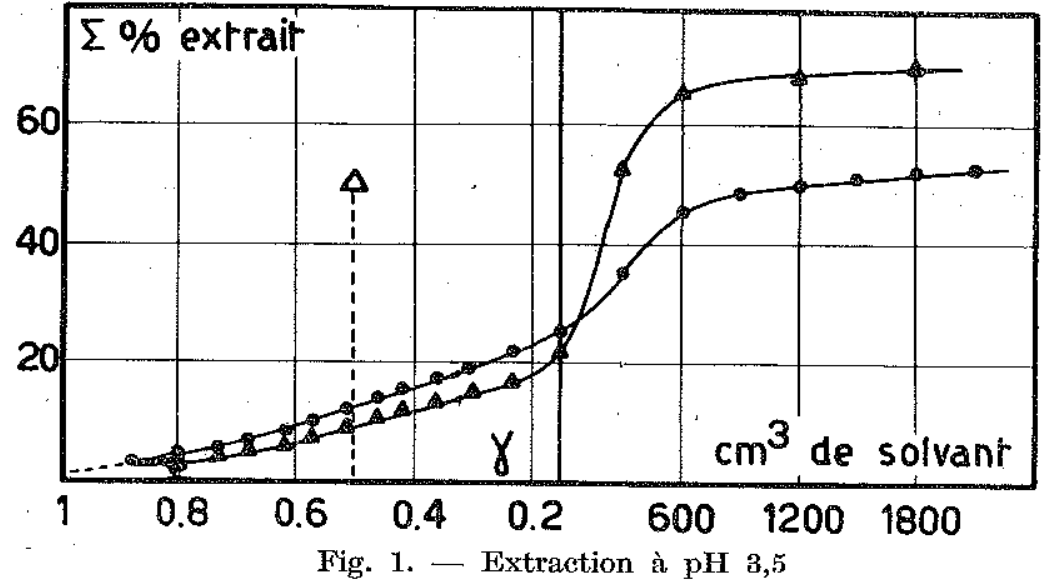

l'alcool méthylique $(40 \%)$ et un tampon acétate de $\mathrm{pH} 3,5$ dont la force ionique de 0,10 , est élevée à 1,5 par addition de $\mathrm{NaCl}$. Les résultats des deux opérations faites respectivement avec une vitesse d'écoulement de $100 \mathrm{cc}$ en $130 \mathrm{mi}$ nutes ( et en 50 minutes (4) sont résumés dans le graphique 1. La somme des pourcentages de produit extrait est portée en fonction $\mathrm{du} \gamma$ de composition du liquide extracteur. L'opération automatique est poursuivie jusqu'au $\gamma$ de 0,15 , valeur pour laquelle environ $25 \%$ de produit sont extraits; on fait ensuite passer au travers de la colonne du solvant pur ce qui entraîne la dissolution de 25 à $50 \%$ de produit. Il subsiste donc dans la colonne une quantité importante d'acide nucléique pratiquement insoluble dans ce tampon acétate de $\mathrm{pH} 3,5$.

Ces extractions fractionnées confirment donc les enseignements tirés des courbes de solubilité qui, nous l'avons rappelé plus haut, révélaient l'existence de fractions d'acide nucléique de solubilité extrêmement faible dans le solvant utilisé.

D'autre part, ces mêmes expériences illustrent une fois de plus l'action solubilisante réciproque des différentes espèces moléculaires constituant l'acide ribonucléique de la levure.

Si nous effectuons une extraction à l'aide d'un seul milieu extracteur de $\gamma$ égal à 0,5 on solubilise $50 \%$ de la 
matière mise en œuvre $(\Delta)$ (voir graphique 1). alors que par extraction continue depuis $\gamma$ égal à 1 jusqu'au $\gamma$ égal à 0,5 on extrait environ $12 \%$ de produit, l'extraction étant cependant pratiquement totale comme nous l'avons vérifié en faisant passer du liquide extracteur de ce $\gamma$ au travers de la colonne jusqu'à dissolution pratiquement nulle.

Dc nombreuses expériences de ce genre ont été faites; toutes conduisent à la même conclusion et confirment les observations antérieures. Rappelons à ce sujet que L. Delcambe ${ }^{1}$ ) a montré par analyse électrophorétique que la fraction $B$ soluble de Chantrenne entrainait de grandes quantités de la fraction A de Chantrenne, dite peu soluble. Il n'est donc pas étonnant que les fractionnements par précipitation soient si difficiles à réaliser.

\section{b) Extraction à $p H 5,5$.}

La durée assez longue d'une extraction au pH 3,5 auquel l'acide pentosenucléique ne semble pas être très stable, pourrait être la cause de transformations de ce produit au cours du fractionnement. Nous avons préféré poursuivre les expériences qui viennent d'être décrites au pH 5,5. A ce $\mathrm{pH}$, l'influence d'une légère modification de composition du mélange sur la solubilité des fractions peu solubles semble encore être exaltée mais on pouvait espérer compenser cette sélectivité plus faible par un meilleur rendement final.

Le graphique 2 résume les résultats obtenus. Le solvant est à nouveau constitué d'un tampon acétate de pH 5,5 et de force ionique 0,10 ; le précipitant est le même tampon dont la force ionique est élevée à 1,5 par addition de $\mathrm{NaCl}$ et contient $40 \%$ d'alcool.

Les courbes ( ) et (o) ont été obtenues en système continu respectivement avec des vitesses d'écoulement de 20 gouttes et de 40 gouttes par minute. La comparaison de ces deux courbes montre qu'à ce $\mathrm{pH}$, l'élimination sélective de produits très solubles est beaucoup plus difficile à réaliser et que nous sommes très loin de l'équilibre lors de l'extrac-

(1) L. Delcambe : Bull. Soc. Chim. Belg., 59, 508 (1950). 
tion. En effet, si avec une vitesse d'écoulement de 40 gouttes par minute on effectue l'extraction de $\gamma: 1,0$ à $\gamma: 0,66$ avec un volume triple de milieu extracteur, on obtient les points de la courbe (o). L'allure des courbes d'extraction dépend donc considérablement des conditions d'extraction à ce $\mathrm{pH}$. Il est par conséquent nécessaire, afin d'obtenir un fractionnement efficace, de conduire l'extraction en système discontinu et de s'assurer à de nombreux $\gamma$ que l'extraction est pratiquement totale.

Une extraction discontinue a donc été réalisée sur $15 \mathrm{grs}$ d'acide nucléique brut dispersé sur 1000 grs de sable à la température de $4^{\circ} \mathrm{C}$ et conduite de façon à obtenir une dizaine de fractions, (intervalle de $\gamma: 0,1$ unité) (courbe $\Delta$ ). L'extraction à chaque $\gamma$ est poursuivie jusqu'au moment où le liquide extracteur ne contient plus qu'environ $20(\gamma)$ de phosphore par cc, la concentration au début de chaque extraction étant de 100 à $150(\gamma)$ de phosphore par cc.

L'extraction est arrêtée lorsque la valeur de $\gamma$ est égale à 0,10 et le résidu de la colonne est dissout par l'eau (fraction 0,0$)$. Cette fraction a été soumise à une nouvelle extraction discontinue à l'aide du même système solvant-précipitant.

La courbe (\$) prouve que cette fraction ne contient plus qu'une faible quantité de produit soluble à des $\gamma$ supérieurs à 0,1 et est considérablement plus homogène que le produit de départ. L'allure de cette courbe montre également que dans les conditions expérimentales adoptées pour ces extractions à pH 5,5, la dégradation est faible et que le processus de fractionnement est efficace.

La nouvelle fraction résiduelle extraite par l'eau pure est désignée par $\gamma: 0,0^{\prime}$.

\section{III. - Caractéristiques PHysico-GHIMIQUes DES FRAC- TIONS OBTENUES.}

L'analyse physico-chimique (diffusion et sédimentation) a été faite sur les fractions de $\gamma: 0,0$ des différentes extractions continues à pH 5,5 et sur toutes les fractions obtenues par extraction discontinue au même $\mathrm{pH}$ (courbe $\Delta$ ) ainsi que sur la fraction $\gamma: 0,0^{\prime}$ (graphique 2). 


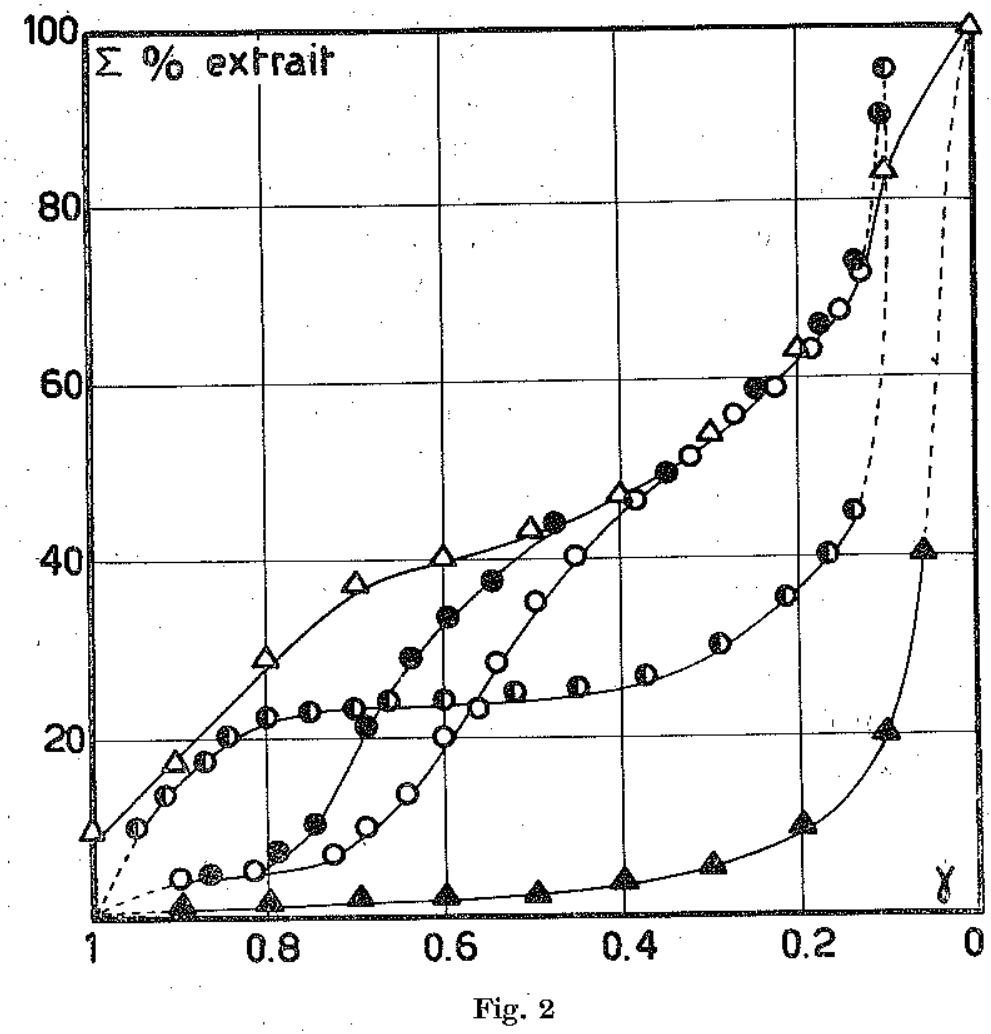

Courbe Extraction continue : 20 gouttes par minute.

O Extraction continue : 40 gouttes par minute.

o Extraction continue : 40 gouttes par minute mais avec un volume triple depuis $\gamma: 1$ jusqu'au $\gamma: 0,66$.

$\Delta$ Extraction discontinue.

Axtraction discontinue effectuée sur la fraction 0,0 .

Dans le tableau I, nous avons comparé les constantes de sédimentation exprimées en unités Svedberg $\left(\mathrm{S}_{20}^{o}\right)$, les constantes de diffusion $\left(\mathrm{D}_{20}^{\circ} \mathrm{cm}^{2} \cdot 10^{-7} / \mathrm{sec}\right)$ et les poids moléculaires déduits de ces deux valeurs pour l'acide pentosenucléique brut de la levure et pour les fractions $\gamma: 0,0$ et $\gamma: 0,0^{\prime}$. Toutes les déterminations ont été faites en tampon acétate de $\mathrm{pH} 5,5$ et de force ionique 0,10 .

Les diffusions ont été faites à $0^{\circ} 4 \mathrm{C}$ dans l'appareil à électrophorèse de Pearson, la concentration variant suivant les expériences de 0,3 à $0,8 \%$. Nous avons vérifié 
qu'une telle variation de concentration ne modifiait la valeur de $D_{20}^{o}$ que de $5 \%$.

Les centrifugations ont été réalisées à l'aide de l'ultracentrifuge Spinco; la concentration en nucléate était de $0,8 \%$ et la vitesse de 59.400 tours par minute

Les valeurs numériques des poids moléculaires n'ont qu'une signification comparative, les échantillons examinés étant manifestement polydispersés et la valeur du volume spécifique partiel adoptée dans les calculs étant incertaines (voir partie expérimentale).

\section{TABLEAU I}

\section{Fractions} $\mathbf{D}_{20}^{\circ}$

$\mathrm{S}_{20}^{\mathrm{o}}$

\begin{tabular}{|c|c|c|c|}
\hline brut & 8,3 & 2,8 & 15.500 \\
\hline$\gamma: 0,0 \Delta$ & 5,1 & 4,1 & 38.000 \\
\hline$-: 0,0$ & 9,2 & 4,1 & 20.500 \\
\hline 一: 0,00 & 7,5 & 4,3 & 27.000 \\
\hline$-: 0,0^{\prime}$ & 4,7 & 4,7 & 47.000 \\
\hline
\end{tabular}

On constate que les fractions $\gamma: 0,0$ se caractérisent par des constantes de sédimentation très voisines mais que la valeur de la constante de diffusion est d'autant plus faible que l'extraction a été mieux conduite $(\Delta<<0)$. Ces constantes de diffusion ont été calculées par la méthode : surface-ordonnée maximum et constituent donc des moyennes se rapprochant de la moyenne en nombre. Nous voyons également que l'échantillon refractionné $(\gamma: 0,0)$ possède une vitesse de sédimentation encore plus élevée et une constante de diffusion plus faible. Il serait par conséquent plus homogène.

Dans le tableau II nous avons condensé les caractéristiques des fractions obtenues par extraction discontinue (graphique 2 ; courbe $\Delta$ ) et à titre de comparaison les mêmes caractéristiques de quelques fractions obtenues par précipitation fractionnée de l'acide brut par le précipitant Chantrenne ( $\mathrm{A}$ et $\mathrm{B}$ ) et de la fraction obtenue après six tours de fractionnement par le précipitant: $\mathrm{CH}_{3} \mathrm{OH} 20 \%$ - tampon acétate de $\mathrm{pH} 3,5$ et $\mu$ égale à $1,7\left(\mathrm{~A}^{\prime}{ }_{1}\right)\left({ }^{1}\right)$.

(1) J.M. GHUYSen : Bull. Soc. Chim. Belg., 59, 490 (1950). 


\section{TABLFAU II}

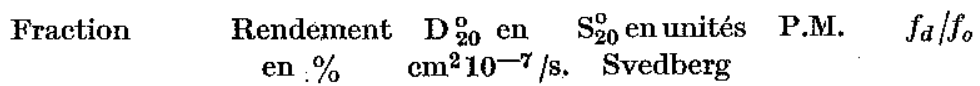

\begin{tabular}{|c|c|c|c|c|c|c|}
\hline \multirow[t]{11}{*}{$Y:$} & 1 & 9,7 & 12,8 & 2,09 & 7.700 & 1,4 \\
\hline & 0,9 & 7,1 & 12,1 & 2,02 & 7.800 & 1,5 \\
\hline & 0,8 & 12,4 & 11,5 & 2,37 & 9.700 & 1,5 \\
\hline & 0,7 & 6,6 & 12,0 & 1,72 & 6.500 & 1,6 \\
\hline & 0,6 & 2,1 & 一 & 一 & - & - \\
\hline & 0,5 & 3,5 & 10,7 & 2,26 & 10.000 & 1,6 \\
\hline & 0,4 & 4,3 & 10,0 & 2,56 & 12.000 & 1,6 \\
\hline & 0,3 & 6,6 & 7,5 & 2,77 & 17.500 & 1,8 \\
\hline & 0,2 & 9,7 & 7,8 & 2,69 & 16.000 & 1,8 \\
\hline & 0,1 & 19,1 & 7,3 & 3,35 & 21.500 & 1,8 \\
\hline & 0,0 & 16,3 & 5,1 & 4,10 & 38.000 & 2,1 \\
\hline & $0,0^{\prime}$ & 40 & 4,7 & 4,66 & 46.500 & 2,1 \\
\hline
\end{tabular}

Précipitation fractionnée par le précipitant Chantrenne (deux tours de fractionnement)

\begin{tabular}{rrrrrr}
\hline A & & & & & \\
B & 35,0 & 10,2 & 2,9 & 13.000 & 1,5 \\
& 45,0 & 19,1 & 1,6 & 4.000 & 1,2 \\
\hline
\end{tabular}

Précipitation fractionnée par le précipitant à l'alcool méthylique (six tours de fractionnement)

\begin{tabular}{llllll}
\hline $\mathbf{A}^{\prime}{ }_{1}$ & 10 & 7,3 & 2,9 & 18.500 & 1,8 \\
\hline
\end{tabular}

Le graphique 3 exprime la variation des poids moléculaires des différentes fractions en fonction du $\gamma$ auquel ces fractions ont été extraitès.

L'examen des valeurs du tableau II et de l'allure de la courbe révèle qu'il est possible d'obtenir par extraction de l'acide pentosenucléique brut de la levure, des fractions de poids moléculaires considérablement plus élevées que ceux 


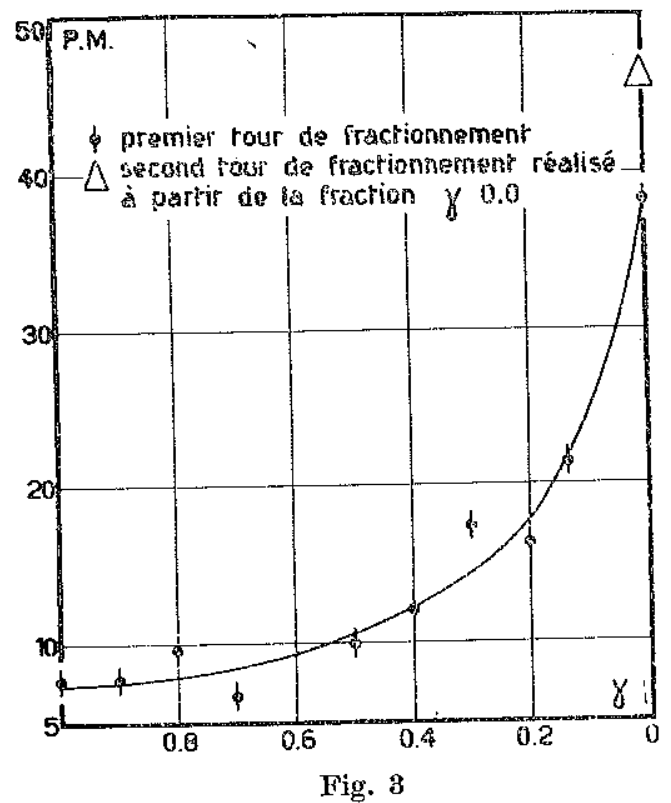

qui caractérisent les échantillons isolés par précipitation fractionnée.

D'autre part, il est manifeste que cet acide tel qu'il a été obtenu, est polydispersé. Seule la technique de l'extraction fractionnée a permis de mettre en évidence, sans aucun doute, cette hétérogénéité de masse moléculaire.

Ce graphique montre également que la variation de poids moléculaire en fonction de la solubilité des différentes fractions ne devient importante que pour les acides extraits à un $\gamma$ inférieur à 0,4 .

Au-dessus de $\gamma 0,4$ on isole donc des fractions de solubilité très différentes mais de poids moléculaire moyen relativement constant.

Cette constatation explique très probablement pourquoi les échantillons d'acide ribonucléique de levure isolés par différents auteurs à l'aide de techniques diverses de précipitation aient des poids moléculaires assez voisins et proches de 10.000 Seuls Cohen et Stanley $\left(^{1}\right)$ ont isolé par décompo-

(1) S.S. Coren et W.M. Stanley : J. Biol. Chem., 144, 589 (1942). 
sition thermique du Virus de la mosaïque du tabac un acide nucléique A qui fraîchement préparé avait un poids moléculaire de 200.000 à 300.000 et qui se décompose spontanément en un acide $B$ plus stable de poids moléculaire 60.000 ; par traitement aux alcalis ces auteurs obtiennent un acide $\mathrm{C}$ de masse moléculaire 15.000 environ.

Notre fraction $\gamma: 0,0^{\prime}$ conservée à l'état sec à basse température est stable pendant plusieurs mois et ne manifeste aucune variation sensible de ses constantes de sédimentation et de diffusion.

Remarquons enfin que les valeurs de ces constantes sont assez proches de celles trouvées par Cohen et Stanley pour leur acide $B$ résultant de la transformation spontanée de l'acide A.

\section{IV. - Conclusions.}

L'acide pentosenucléique de la levure tel qu'il est généralement préparé est hétérogène et seule la technique de fractionnement par extraction systématique a permis l'isolement de fractions d'un poids moléculaire élevé.

Le produit obtenu par Volkin et Carter estatrès probablement également polydispersé et dégradé, les constantes de sédimentation données par ces auteurs étant de même ordre de grandeur que celles de notre produit purifié par précipitation fractionnée.

Nous croyons donc que le problème de l'isolement d'un acide pentosenucléique peu ou pas dégradé ne pourra être résolu qu'en partant de nouvelles bases (*).

\section{V. - Partie expérimentale.}

I) Extraction fractionnée

a) Préparation de la colonne

La substance inerte utilisée pour obtenir une dispersion élevée

(*) Ce travail était en cours d'impression lorsque nous avons eu connaissance de l'article de E.L. Grinnan et W.A. Mosher (J. Biol. Chem., 191, 719 (1951)) qui, en combinant.l'inactivation par le chlorhydrate de guanidine, la dénaturation par la chaleur et l'élimination des protéines par le chloroforme ont obtenu un acide nucléique à partir du foie de constante de sédimentation de 10,4, valeur donc encore supérieure à celle trouvée par Cohen et Stanley.

de l'ari et tami solution sières $e$ filtre damme Le nuc versée et sèclié gène, 0 dans $n$ sable e refroid ques ci

quelqu un fort les par reste o du com matiqu sont cor à capte La piy entière 
de l'acide nucléique est constituée par du sable lavé et calciné, moulu et tamisé à 100 mesh. Après ce traitement le sable est traité avec une solution de détergent afin de mettre en suspension toutes les poussières et les très fines particules de sable qui passeraient au travers du filtre en verre frité de la colonne. Après décantation, on lave abondamment le sable avec de l'eau distillée et de l'alcool; on le sèche ensuite. Le nucléate est dissous dans le minimum d'eau et cette solution est versée sur le sable; l'ensemble est homogénéisé soigneusement au mortier et sèché en couches minces. Afin de s'assurer que la dispersion est homogène, on prélève de petits échantillons de sable que l'on met en suspension dans un volume d'eau connu. On mesure l'absorption à $258 \mathrm{~m} \mu$. Le sable est enfin introduit par petites portions dans la colonne en verre refroidie; l'élimination de l'air occlus est obtenu par addition de quelques ce de précipitant froid après chaque introduction de sable.

\section{b) Conduite de l'opération}

L'opération en continu est conduite comme décrit précédemment; quelques modifications techniques ont été introduites afin d'obtenir un fonctionnement plus régulier de l'appareil. La figure 4 représente les parties $\mathbf{B}$ et $\mathbf{D}$ de l'appareil à extraction micro décrit précédemment.

En (a) nous avons disposé une petite soupape magnétique qui reste ouverte pendant toute la durée de l'opération. En cas de coupure du courant électrique la valve se ferme et toute l'opération est automatiquement arrêtée. Les tubes d'amenée des liquides extracteurs (b) sont coudés et portent à la partie supérieure un petit réservoir (c) destiné à capter les éventuelles bulles d'air qui tendent à désamorcer l'appareil. La pipette $\mathrm{D}$ a également été modifiée comme schématisé et donne entière satisfaction.

\section{II) Volume spécifique partiel apparent}

Cette détermination a été faite à partir d'une solution de nucléate à $2 \%$ en tampon acétate de $\mathrm{pH} 5,5$ et de force ionique de 0,10 à $25^{\circ} \mathrm{C}$. Le taux d'humidité a été déterminé par chauffage sous vide à différentes températures $\left(105^{\circ} ; 115^{\circ} \mathrm{C} ; 140^{\circ}\right)$ chaque fois jusqu'à poids apparemment constant. Ce taux n'avait pas même valeur dans chaque essai $(11,3 \%$ à $13,0 \%)$ et il est done difficile de fixer exactement la valeur de ce volume spécifique partiel. Nous avons trouvé pour trois échantillons :

$\begin{array}{ll}\text { ribonucléate brut : } & 0,48 \mathrm{cc} / \mathrm{gr} \\ \text { fraction } \gamma: 0, \gamma & 0,50 \mathrm{cc} / \mathrm{gr} \\ \text { fraction } \gamma: 0,1 & 0,50 \mathrm{cc} / \mathrm{gr}\end{array}$

Précédemment L. Delcambe et V. Desseux $\left({ }^{1}\right)$ avaient obtenu $0,53 \mathrm{cc} / \mathrm{gr}$. Signalons la valeur de 0,578 obtenue par Cohen et Stanley

(1) L. Delcambe et V. Desreux : Bull. Soc. Chim. Belg., 59, 521 (1950) 


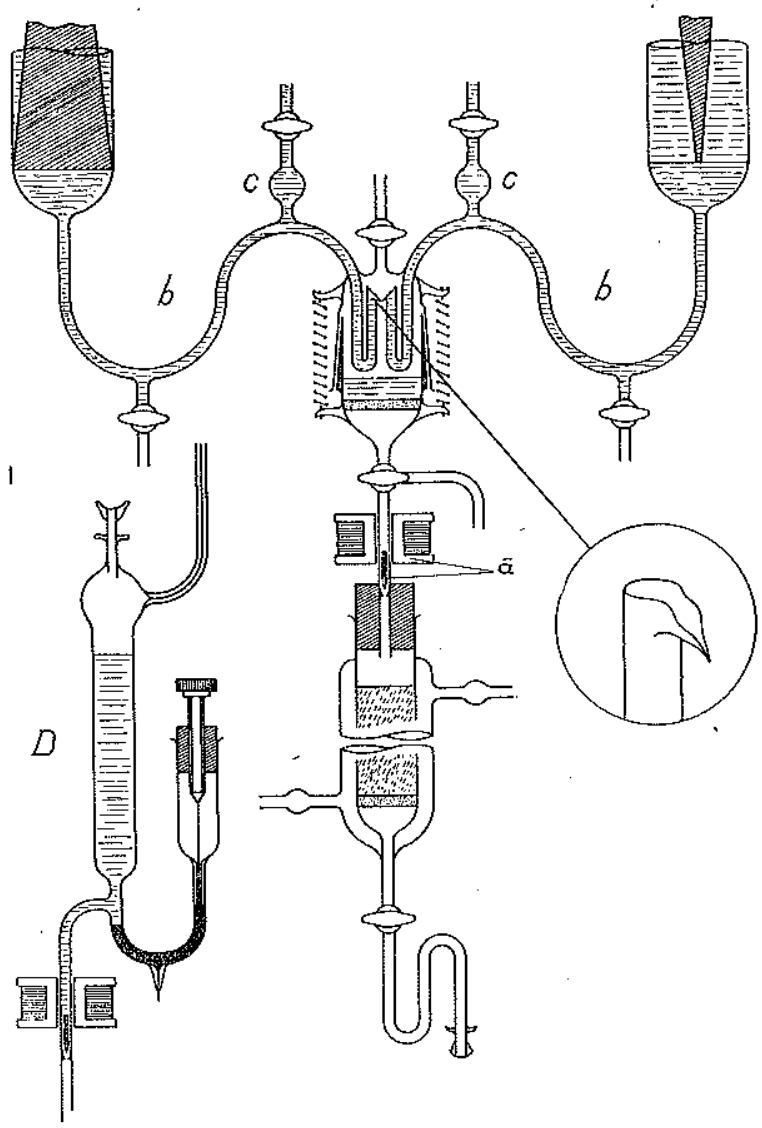

ainsi

lisati

Fig. 4

(1) pour l'acide ribonucléique du virus de la mosäque du tabac, et la valeur de 0,48 par l'acide de levure obtenue par Bacher et Allen $\left({ }^{2}\right)$. C'est cette valeur que nous utilisons dans les calculs précédents.

Cette étude a été subsidiée par le Centre National de Biologie Physico-chimique, par le Centre de Recherches pour. la Pénicilline et les autres Antibiotiques et par la Fondation Rockefeller.

Les diffusions ont été faites à l'aide de l'appareil mis à la disposition du Laboratoire de chimie-physique par le Fonds National de la Recherche Scientifique.

(1) S.S. Cohen et W.M. StanLey : J. Biol. Chem., 144, 589 (1942).

(2) J.E. BACher et E.W. Auten : J. Biol. Chem., 184, 511 (1950). 
Nous tenons à remercier ces organismes pour leur appui, ainsi que I'I. R. S. I. A. pour l'octroi d'une bourse de spécialisation à l'un de nous (J. G.).

UNIVERSITÉ DE LIÈGE

Laboratoire de Chimie physique. 